\title{
Obtención de partículas de óxido de cobre a bajo costo, para ser aplicadas en medio acuoso contaminado.
}

\section{Obtaining copper oxide particles at low cost to be applied in contaminated aqueous medium.}

\section{Obtenção de partículas de óxido de cobre a baixo custo para ser aplicadas no meio aquoso contaminado}

\author{
Miryam Rincón-Joya ${ }^{1}$, Angela Mercedes Raba-Páez², José Barba-Ortega ${ }^{3}$
}

Forma de citar: M. R. Joya, A. M. Raba-Páez, J. Barba-Ortega, "Obtención de partículas de óxido de cobre a bajo costo, para ser aplicadas en medio acuoso contaminado", Respuestas, vol. 21, no. 1, pp. 89-96, 2016.

Recibido:

Agosto 18 de 2015

Aceptado:

Noviembre 10

de 2015

${ }^{1}$ Postdoctorado en física mrinconj@unal.edu.co Orcid: 0000-0002-4209-16

Universidad Nacional de Colombia

Facultad de Ciencias, Departamento de Física

Bogotá-Colombia

${ }^{2}$ Maestría en Física Email: angelamercedesrp@ufps.

edu.co

Orcid: 0000-0001-8147-6323 Universidad Francisco de Paula Santander

Departamento de Física

Cúcuta-Colombia

${ }^{3}$ Doctor en Física jjbarbao@unal.edu.co Orcid: 0000-0003-3415-1811

Universidad Nacional de Colombia

Facultad de Ciencias, Departamento de Física Bogotá-Colombia

\section{Resumen}

Objetivo: En este artículo se presenta una metodología en la preparación de partículas de óxido de cobre $(\mathrm{CuO})$ por métodos a bajo costo de preparación como son precursores poliméricos y evaporación de solventes, visando ser aplicadas en medios acuosos contaminados. Método: las muestras fueron preparas por diferentes rutas de síntesis y sometidas a diferentes temperaturas de calcinación. Resultados:Las muestras fueron obtenidas por ruta 1: método Pechini (precursores poliméricos) modificado, cambiando concentraciones y con adición de $\mathrm{HNO}_{3}$ y $\mathrm{NH}_{4} \mathrm{OH}$ para regular $\mathrm{pH}$, y la ruta 3: método Pechini sin regular pH. La calcinación permitió obtener muestras de $\mathrm{CuO}$ en la estructura monoclínica y dependiendo del método cambia la morfología de las mismas. Conclusión: La ruta 3 o método sin regular el $\mathrm{pH}$ fue el que presento mejores resultados, se obtuvo la estructura monoclínica y el tamaño de partícula es menor, lo cual permite predecir las consecuencias en el desempeño fotocatalítico en la degradación de contaminantes es posiblemente bueno.

Palabras clave: (óxido de cobre, precursores poliméricos, rayos X, morfología de las muestras)
Abstract
Objective: This paper presents a methodology in the preparation of particles of copper oxide $(\mathrm{CuO})$ by low-cost methods of preparation, such as polymeric precursors and solvent evaporation, aiming to be applied in contaminated aqueous media. Method: the samples were prepared by different routes of synthesis and subjected to different calcination temperatures. Results: Samples were obtained by Route 1: modified Pechini method (polymeric precursors), changing concentrations and with addition of $\mathrm{HNO} 3$ and $\mathrm{NH} 4 \mathrm{OH}$ to regulate $\mathrm{pH}$, and Route 3: Pechini method without $\mathrm{pH}$ regulation. Calcination allowed to obtain $\mathrm{CuO}$ samples in the monoclinic structure and morphology changes depending on the method. Conclusion: Route 3 or Pechini method without regulating the $\mathrm{pH}$ showed the best results with monoclinic structure and the lower particle size, which allows to predict a good photocatalytic performance in the degradation of pollutants. 
No. 1

Enero - Junio 2016 ISSN 0122-820X

E-ISSN 2422-5053

\section{Introducción}

El estudio del óxido de cobre es de gran importancia ya que sus propiedades físicoquímicas hacen de él un material promisorio en aplicaciones fotocatalíticas [1], [2], [3]. Los óxidos de cobre son compuestos inorgánicos [4] con fórmulas $\mathrm{Cu}_{2} \mathrm{O}$ para el óxido cuproso u óxido de cobre (I) y $\mathrm{CuO}$ para el óxido cúprico u óxido de cobre (II) [5]. Expuesto al aire, se forma primero $\mathrm{Cu}_{2} \mathrm{O}$ y después óxido cúprico $(\mathrm{CuO})$ [6].

Se ha reportado la síntesis de nanocristales de $\mathrm{Cu}_{2} \mathrm{O}$ [7] modulando la morfología, la temperatura, el ambiente libre, el uso de surfactantes y la dependencia de la actividad catalítica orgánica con la forma de las nanopartículas [8]. En este trabajo desarrollan una ruta química libre de surfactantes para fabricar nanotubos, octaedros, esferas, placas y poliedros de $\mathrm{Cu}_{2} \mathrm{O}$, mediante la variación de la atmósfera de reacción (aire y argón) y del agente reductor [8], [9]. Aparentemente la adsorción de oxígeno y reacciones en la superficie del $\mathrm{Cu}_{2} \mathrm{O}$ pueden ser capaces de frenar la reducción de $\mathrm{Cu}(\mathrm{OH})_{2}$ y la nucleación

de $\mathrm{Cu}_{2} \mathrm{O}$ y además ejercer una influencia notable en el crecimiento[10].

Estudios que emplean el $\mathrm{CuO}$ como fotocatalizador generalmente aplican este material en la forma micrométrica, obtenido a través de una simple calcinación del óxido comercial o a través de tratamientos con reactivoscomoelperóxidodehidrógeno,ácidos y bases [11]. Esto resulta ser un inconveniente para la fotocatálisis heterogénea ya que éste fenómeno es superficial dependiente en su mayoría del área superficial, de los grupos superficiales adsorbidos (tales como $\mathrm{OH}-$ ) [12] y por lo tanto dependiente también del tamaño de partícula obtenido.

La fotocatálisis heterogénea (FH) [13] constituye el proceso fotocatalítico que tiene lugar en la frontera interfacial entre dos fases. En general es una tecnología avanzada de oxidación (TAO), caracterizadas por la generación in situ de especias altamente oxidantes, como por ejemplo radicales $\mathrm{OH}$ [14]. 
En la $\mathrm{FH}$ se han empleado óxidos semiconductores ya que presentan las siguientes ventajas:

- Bajo costo.

- Inocuidad.

- No presentan una pérdida sustancial de la actividad fotocatalítica.

- Existe la posibilidad de recuperar el sólido mediante procesos sencillos como filtrado o centrifugado, permitiendo su uso en varios ciclos.

Se ha reportado que nanocompuestos a base de cobre han sido empleados en procesos catalíticos, para la reducción de NOx y para la combustión de hidrocarburos [15].

Es relevante el desarrollo de este trabajo ya que permite demostrar que el tamaño de las partículas de $\mathrm{CuO}$ se puede modular cambiando el valor del $\mathrm{pH}$ de la solución de reacción o la concentración inicial de precursores o inclusive mediante la adición de solventes de alta viscosidad. El tamaño de partícula también se modifica por la temperatura de calcinación de las muestras. Para este estudio se usó un método de bajo costo llamado de precursores poliméricos o método de pechini.

\section{Materiales y Métodos}

El polvo se obtuvo por el método Pechini. Inicialmente se preparó una solución acuosa $20 \%$ de cloruro cúprico dihidratado (CuCl2.2H2O- Panreac $99.8 \%$ ) por masa, que contiene $23.3518 \mathrm{~g}$ de soluto. Por separado se preparó una solución acuosa $20 \%$ de ácido cítrico monohidratado (Panreac 99.8\%) por masa, que contiene $88.1285 \mathrm{~g}$ de él. Se mezclaron las dos soluciones en agitación constante manteniéndose una temperatura de $80^{\circ} \mathrm{C}$ durante media hora. A la mezcla se le adicionó $52.7873 \mathrm{ml}$ de etilenglicol (Panreac $99.8 \%$ ) a $90^{\circ} \mathrm{C}$; se mantuvo esta temperatura hasta que se redujo el volumen de agua. La resina obtenida se pre-calcinó a $300^{\circ} \mathrm{C}$, a $150^{\circ} \mathrm{C}$ y finalmente a $300^{\circ} \mathrm{C}$, con el fin de eliminar solventes aún presentes, y el sólido resultante de estas pre-calcinaciones se maceró utilizando un mortero de ágata. El material resultante se tamizó inicialmente en tamiz No. 20 (tamaño de poro $850 \mu \mathrm{m}$ ) y luego en tamiz No. 50 (tamaño de poro 300 $\mu \mathrm{m})$. Los polvos obtenidos del tamizaje se sometieron a los tratamientos térmicos finales.

En el desenvolvimiento de la siguiente investigación se tuvieron en cuenta algunos puntos claves para obtener las muestras. Las características de los métodos seguidos en la obtención de las muestras fueron las Siguientes:

- Caracterizar los materiales obtenidos mediante difracción de rayos $\mathrm{X}$ y microscopía de barrido electrónico con el fin de comparar las propiedades físicoquímicas de los óxidos obtenidos.

- Establecer por cuál de los dos métodos de síntesis empleados se puede inferir un tamaño de partícula deseable para aplicaciones fotocatalíticas.

- Verificar que para fines fotocatalíticos los materiales calcinados a mayor temperatura podrían exhibir una mayor actividad fotocatalítica.

\subsection{Rutas de Síntesis}

En el método de pechini inicialmente se calienta etilenglicol a $70^{\circ} \mathrm{C}$ y se adicionó lentamente de ácido cítrico manteniendo constante la temperatura del sistema a $70^{\circ} \mathrm{C}$, y agitando continuamente la mezcla, hasta obtener una solución transparente. Por separado se prepara la solución del precursor a $0,3 \mathrm{M}$ de cloruro cúprico en una solución acuosa de $0.1 \mathrm{M}$ de ácido nítrico. A la mezcla de etilenglicol y ácido cítrico se le agrega la solución del precursor de cobre y luego se reduce la temperatura a $25^{\circ} \mathrm{C}$ para comenzar a adicionar hidróxido de amonio hasta llevar
Enero - Junio 2016 ISSN 0122-820X

E-ISSN 2422-5053 PP: 89-96 
No. 1

Enero - Junio 2016 ISSN 0122-820X E-ISSN 2422-5053 la solución a un $\mathrm{pH}$ de 4.5. Garantizando que no se forme ningún precipitado en el sistema; la solución se calienta a $90^{\circ} \mathrm{C}$, en agitación constante, hasta que se forma una resina. $\mathrm{La}$ resina obtenida se pre-calcina a $300^{\circ} \mathrm{C}$ y el sólido resultante de esta pre-calcinación se macera utilizando un mortero de ágata. Los polvos obtenidos se someten a tratamientos térmicos finales [16].

El método de evaporación de solventes puede ser empleado con sales o nitratos como precursores de partida. El solvente que se emplea en este método depende de la solubilidad del precursor y puede ser agua o etanol. En cuanto a la cantidad de éste depende del grado de solubilidad del precursor, la cual se beneficia empleando una agitación contante. Posterior a la preparación de la solución de partida, la cual contiene los cationes de interés, se evapora el solvente en estufa, y el reducto se pone en crisol para llevarlo a mufla, y así garantizar la completa evaporación de compuestos orgánicos. Finalmente el polvo resultante se lleva temperaturas de análisis finales. En general en éste método se producen polvos sin emplear procesos de desaglomeración. Este método en la investigación está actualmente en caracterización inicial para poder hacer una comparación con el método pechini.

En este trabajo se empleó el método de pechini utilizando la ruta 1 (R1) y la ruta $3(\mathrm{R} 3)$.

Estos métodos se describen de una forma resumida a continuación. De una forma extensa y para otros materiales se encuentran en otros trabajos de la literatura [16].

R1 Ruta 1:método Pechini modificado, cambiando concentraciones y con adición de $\mathrm{HNO}_{3}$ y $\mathrm{NH}_{4} \mathrm{OH}$ para regular $\mathrm{pH}$.

R3 Ruta 3:método Pechini sin regular $\mathrm{pH}$.

\section{Resultados y análisis $3.1 \mathrm{Cu}_{2} \mathrm{O}$ sintetizado vía ruta $\mathrm{R} 1$}

R1 CuPre: muestra precalcinada a $300^{\circ} \mathrm{C}, 4 \mathrm{~h}$ en ascenso $3 \mathrm{~h}$ en meseta.

Una vez se tiene el material pre-calcinado se somete a diferentes temperaturas de calcinación. En la calcinación se obtuvo la fase estructural monoclínica deseada.

En la tabla I se observan las temperaturas de calcinación de las muestras, el tiempo de ascenso y el tiempo en mesta durante el proceso de la obtención de las muestras. La muestra a $650^{\circ} \mathrm{C}$ se dejó menos tiempo en ascenso y en meseta, en este caso el material se estaba evaporando y no quedaba buena cantidad de muestra para su respectiva caracterización, la perdida de muestra era extremadamente alta. En el caso de la muestra a $550^{\circ} \mathrm{C}$ la explicación del tiempo en meseta es similar a la muestra de $650^{\circ} \mathrm{C}$.

Tabla I. Evolución con la temperatura de la estructura cristalina del óxido de cobre $(\mathrm{CuO})$ sintetizado por la ruta $\mathrm{R} 1$.

$\begin{array}{llcc}\text { Muestra } & \mathrm{T}\left({ }^{\circ} \mathrm{C}\right) & \text { Tiempo ascenso } & \begin{array}{c}\text { Tiempo } \\ \text { meseta }\end{array} \\ \text { R1Cu250 } & 250 & 2 \mathrm{~h} & 2 \mathrm{~h} \\ \mathrm{R} 1 \mathrm{Cu} 350 & 350 & 2 \mathrm{~h} & 2 \mathrm{~h} \\ \mathrm{R} 1 \mathrm{Cu} 450 & 450 & 2 \mathrm{~h} & 2 \mathrm{~h} \\ \mathrm{R} 1 \mathrm{Cu} 550 & 550 & 2 \mathrm{~h} & 1 \mathrm{~h} \\ \mathrm{R} 1 \mathrm{Cu} 650 & 650 & 40 \mathrm{~min} . & 15 \mathrm{~min}\end{array}$

Fuente: autores

En la figura 1 los patrones de difracción asociados a las muestras R1CuPre, R1Cu250 y R1Cu350 muestran la presencia de cloruro de cobre hidratado. Los difractogramas de las muestras R1Cu450, R1Cu550 y R1Cu650 se relaciona con el del $\mathrm{CuO}$ (sistema cristalino monoclínico). Como se puede observar en la figura 1 en la calcinación de $550^{\circ} \mathrm{C}$ y $650^{\circ} \mathrm{C}$ aparecen en los difractogramas de rayos $\mathrm{x}$ los principales picos de la estructura monoclínica. Para las otras temperaturas de calcinación no hay definida una estructura específicamente. 


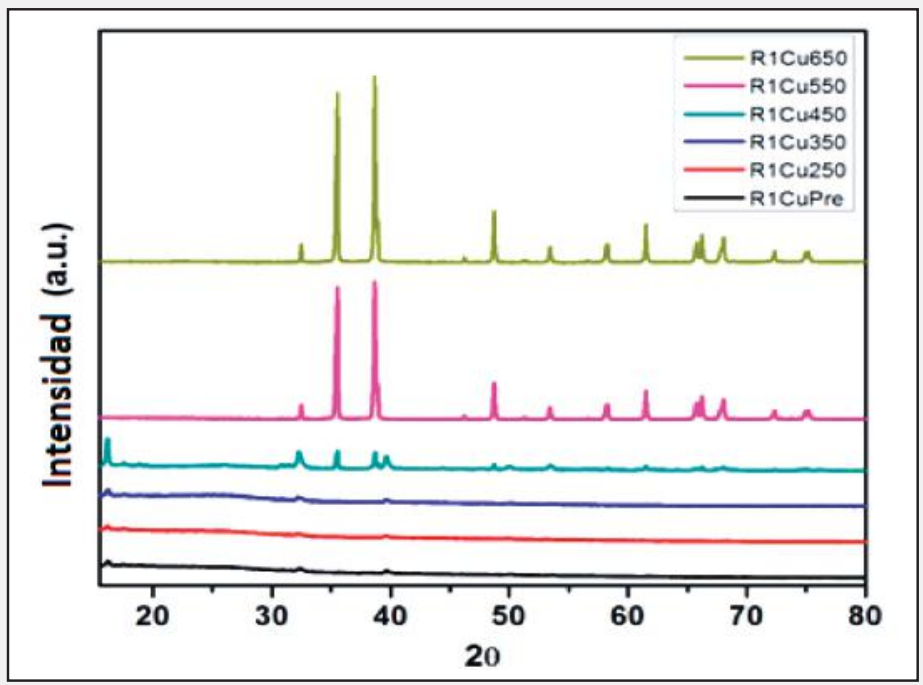

Figura 1. Evolución con la temperatura de la estructura cristalina del óxido de cobre $(\mathrm{CuO})$ sintetizado por la ruta $\mathrm{R} 1$.

Para el CuO los planos principales son: (hkl), (2): (110) (32,3); (002), (35.5); (111),(38.7); (-202), (48.7); (202), (58,2);(-113),(62,4) y $(-311),(68,4)$.

Fuente: autores

En la tabla II se presenta a modo de ejemplo la evolución de la temperatura de calcinación para el óxido de cobre por el método R3. En este caso recordemos que no se hizo cambio del $\mathrm{pH}$. El tiempo de ascenso y tiempo en meseta para la preparación de las muestras fue exactamente el mismo (2h), este no cambio, en el caso de las muestras método R1 si cambio. En este caso la evaporación de material con la temperatura de calcinación fue mejor, indicando poca perdida de muestra durante el proceso.
Tabla II. Evolución con la temperatura de la estructura cristalina del óxido de cobre $(\mathrm{CuO})$ sintetizado por la ruta $\mathrm{R} 3$.

\begin{tabular}{lccr}
\hline Muestra & $\mathrm{T}\left({ }^{\circ} \mathrm{C}\right)$ & Tiempo ascenso & $\begin{array}{r}\text { Tiempo } \\
\text { meseta }\end{array}$ \\
R3Cu350 & 350 & $2 \mathrm{~h}$ & $2 \mathrm{~h}$ \\
$\mathrm{R} 3 \mathrm{Cu} 450$ & 450 & $2 \mathrm{~h}$ & $2 \mathrm{~h}$ \\
$\mathrm{R} 3 \mathrm{Cu} 550$ & 550 & $2 \mathrm{~h}$ & $2 \mathrm{~h}$ \\
\hline
\end{tabular}

Fuente: autores

En la figura 2 el patrón de difracción asociado a la muestra R3CuPre muestra la presencia de cloruro de cobre hidratado y zinc; el difractograma de la muestra $\mathrm{R} 3 \mathrm{Cu} 350$

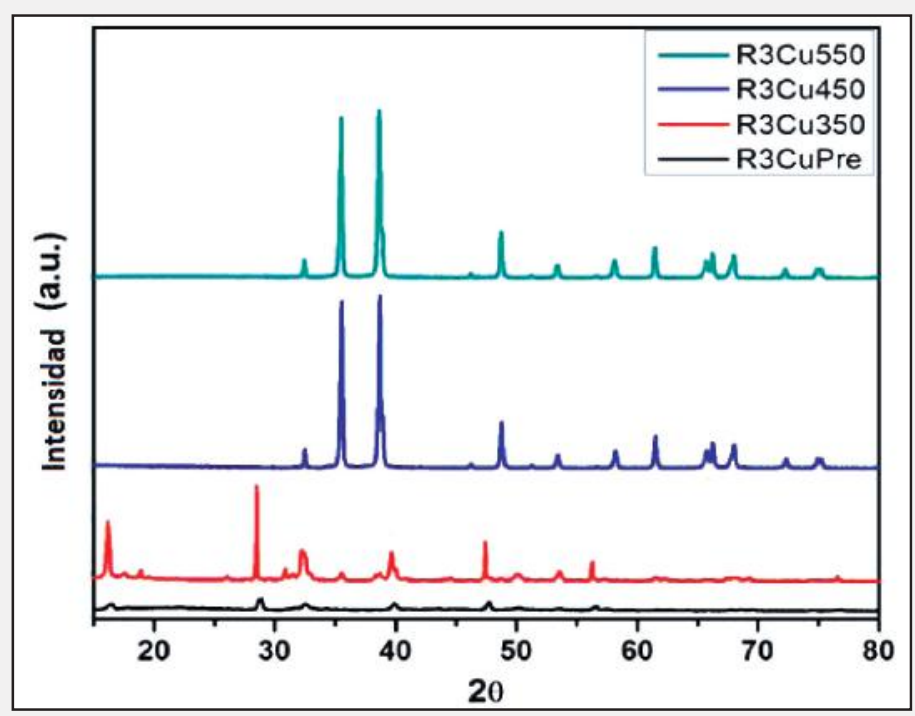

Figura 2. Evolución con la temperatura de la estructura cristalina del óxido de cobre $(\mathrm{CuO})$ sintetizado por la ruta $\mathrm{R} 3$. Para el $\mathrm{CuO}$ los planos principales son : (hkl ), (2): (110) (32,3); (002), (35.5); (111),(38.7); (-202), (48.7); (202), $(58,2) ;(-113),(62,4)$ y $(-311),(68,4)$.

Fuente:autores 
No. 1

Enero - Junio 2016 ISSN 0122-820X E-ISSN 2422-5053

PP: 89-96 muestra la presencia de cloruro de cobre hidratado. Los difractogramas de las muestras $\mathrm{R} 3 \mathrm{Cu} 450$ y $\mathrm{R} 3 \mathrm{Cu} 550$ se relacionan con el del $\mathrm{CuO}$ (sistema cristalino monoclínico). Como se observa en la figura 2 el método R3 permite la obtención de la fase a temperaturas más bajas de calcinación.

En la figura 3 se presenta una comparación entre los difractogramas obtenidos a las mismas temperaturas pero para muestras sintetizadas con diferente método (R1 y R3). Se observa que por las dos rutas no se alcanza

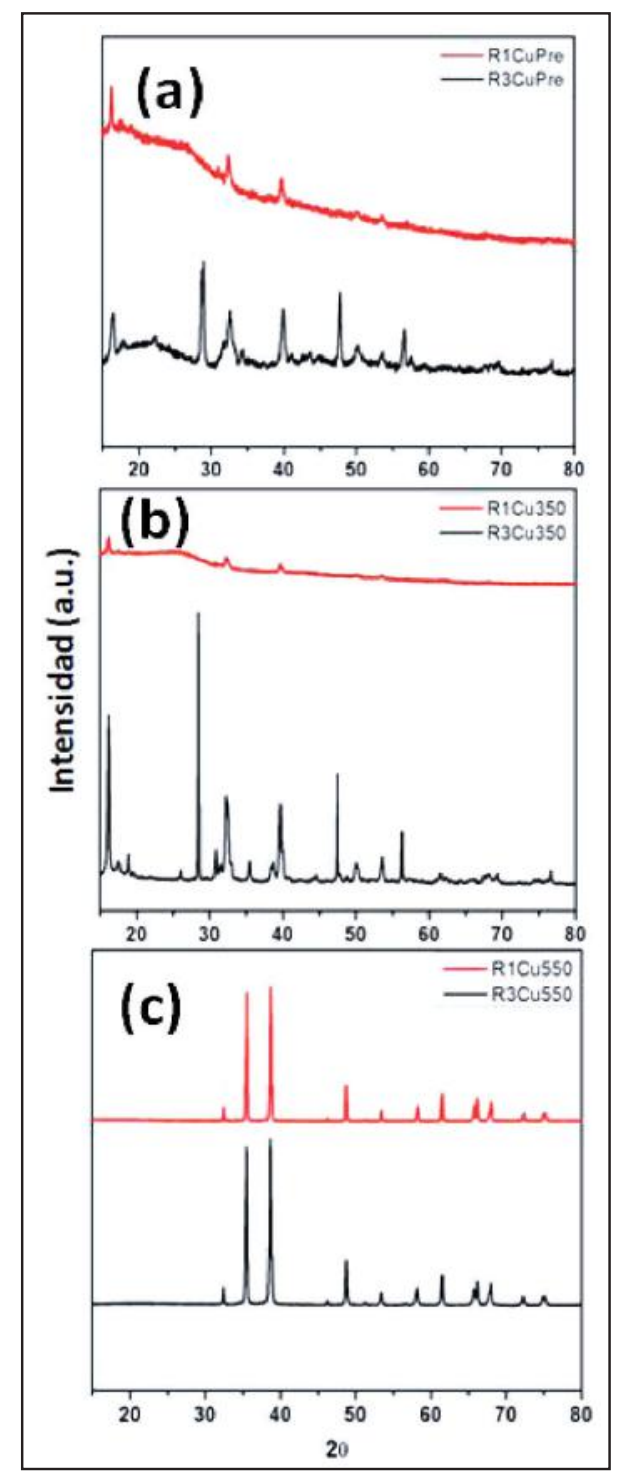

Figura 3. Comparación de la estructura cristalina del óxido de cobre $(\mathrm{CuO})$ sintetizado por las rutas $\mathrm{R} 1$ y R3 en diferentes temperaturas. Para el $\mathrm{CuO}$ los planos principales son: (hkl), (2): (110) $(32,3)$; (002), (35.5); (111),(38.7); (-202), (48.7); (202), $(58,2) ;(-113),(62,4)$ y $(-311),(68,4)$. Fuente: autores el óxido deseado únicamente empleando la precalcinación. A $350^{\circ} \mathrm{C}$ aún se observa mayoritariamente la presencia de cloruro de cobre hidratado. A $450^{\circ} \mathrm{C}$ y $550^{\circ} \mathrm{C}$ se logra alcanzar el sistema $\mathrm{CuO}$ (sistema cristalino monoclínico) por ambos métodos. En la figura 3 (a) el óxido precalcinado. Figura 3 (b) $350^{\circ} \mathrm{C}$, como se observa en (a) y (b) el difractograma es más definido para el método 3. En la figura 3 (c) en $550^{\circ} \mathrm{C}$ las muestras por los dos métodos ya están en la estructura monoclínica.

En la morfología por microscopía electrónica de barrido (MEB) las imágenes muestran una diferencia significativa entre los dos métodos de preparación de las muestras, figura4. En la figura 4 (a) corresponde a la muestra preparada por el método R3 a una calcinación de $800^{\circ} \mathrm{C}$. La figura 4 (b) se observa la figura MEB para la muestra a $800^{\circ} \mathrm{C}$ por el método R1. En la figura 4 (c), (d) corresponden al método R3 y $\mathrm{R} 1$ para una temperatura de $650^{\circ} \mathrm{C}$. La figura 4 (e), (f) corresponden a muestras por el método R3 y R1 a una temperatura de $550^{\circ} \mathrm{C}$ de calcinación.

Como se observa en la figura 4 las partículas son más difinidas por el método R3, para este caso la barra d referencia es de $1 \mu \mathrm{m}$ y para R1 de $2 \mu \mathrm{m}$. Esto nos indica que las muestras obtenidas por el método R3 son las que posiblemente tengan mejor desempeño fotocatalitico. Las partículas son pequeñas y bien definidas. En el caso del Método R1 es posible que no presente respuesta alguna en la degradación de material contaminante, debido al tamaño de partícula. El tamaño de partícula es importante en los efectos fotocatalitico según la literatura [2]. Otro efecto que se puede observar en la figura 4 es que sin importar el método para temperaturas muy elevadas como $800^{\circ} \mathrm{C}$, el tamaño de partícula comienza a crecer, lo que indica que temperaturas muy elevadas tampoco son apropiadas para formar muestras de $\mathrm{CuO}$ para degradación. 


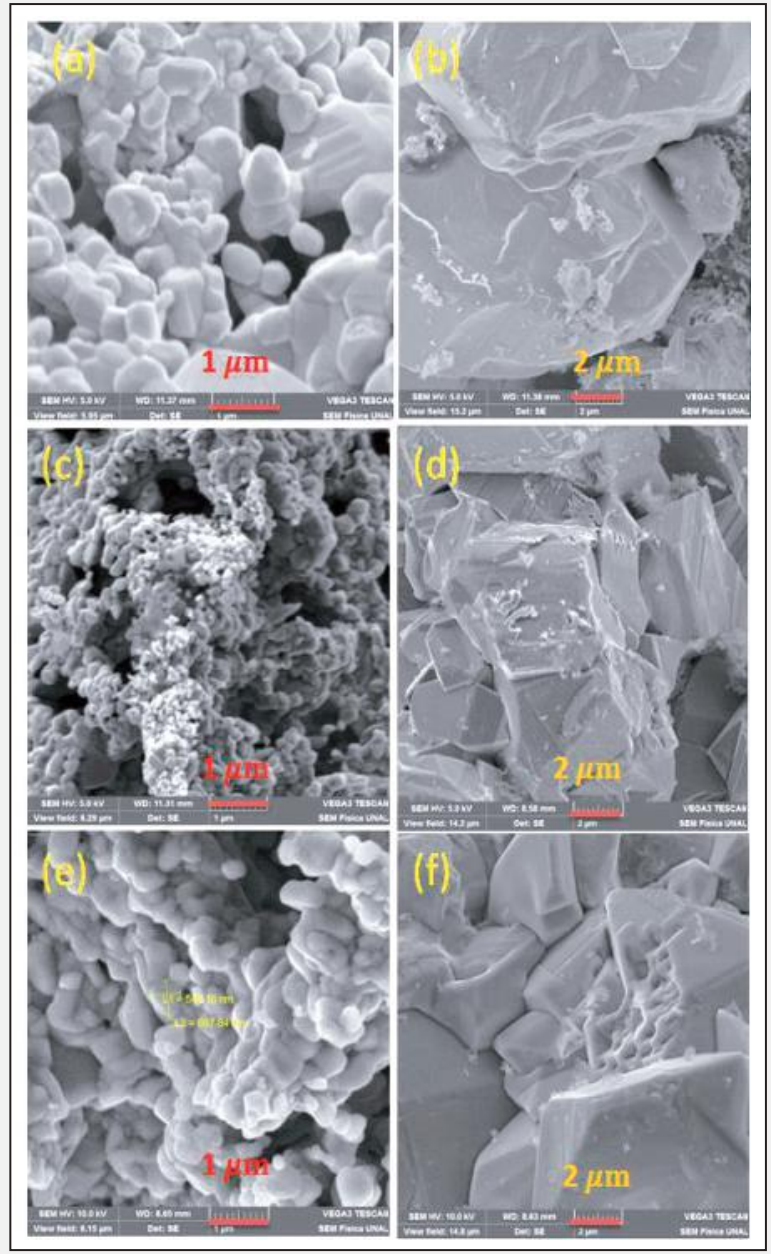

Figura 4. Comparación de la estructura MEB del óxido de cobre $(\mathrm{CuO})$ sintetizado por las rutas $\mathrm{R} 1$ y $\mathrm{R} 3$ en diferentes temperaturas; (a) $800^{\circ} \mathrm{C}$ ruta $\mathrm{R} 3$, (b) $800^{\circ} \mathrm{C}$ ruta $\mathrm{R} 1$, (c) y (d) $650^{\circ} \mathrm{C}$ para ruta $\mathrm{R} 3$ y R1, (e) y (f) $550^{\circ} \mathrm{C}$ método $\mathrm{R} 3$ y $\mathrm{R} 1$ respectivamente. Fuente: autores

\section{Conclusiones}

En el presente trabajo demostramos la formación de partículas $\mathrm{CuO}$ a una temperatura de $550^{\circ} \mathrm{C}$ por los dos métodos de crecimiento de las muestras R1 y R3 con estructura monoclinica. Como se observa en la morfología de las muestras la obtención de partículas definidas y de menor tamaño se consigue por el método R3. Las muestras obtenidas por el método R3, por su forma y tamaño de las partículas prometen ser muestras de buena calidad para ser usadas en actividad fotocatalitica, éste método es de bajo costo en comparación con otros métodos de preparación de muestras encontrados en la literatura.

\section{Agradecimientos}

Agradecimientos especial a la Universidad Francisco de Paula Santander Cúcuta, Colombia y Universidad Nacional de Colombia departamento de Física Bogotá por el apoyo dado en el desenvolvimiento de esta investigación.

\section{Referencias}

[1] A. Ang1, D. Sanl1, C. Erkey, Ö. Birer. "Catalytic activityof copper (II) oxide prepared via ultrasound assisted Fenton-like reaction", Ultrasonics Sonochemistry, vol. 21, no. 2, pp. 854859, 2014.

[2] R. G Zhou, Y. Zheng, D.A HulicovaJurcakova, y Z.G Qiao, "Enhanced electrochemical catalytic activity by copper oxide grown on nitrogen-doped reduced graphene oxide", Journal of Materials Chemistry A, vol. 42, pp. 13179- 13185, 2013.

[3] Y. Ukisu, S. Sato, G. Muramatsu, K. Yoshida. "Activity enhancement of copper-containing oxide catalysts by addition of cesium in the reduction of nitric oxide", Catalysis Letters, vol. 16, no. 1, pp. 11-16, 1992.

[4] C. Petit, J. Burress, T. J. Bandosz. "The synthesis and characterization of copper-based metal-organic framework/graphite oxide composites", Carbon, vol. 49, no. 2, pp. 563-572, 2011.

[5] J. P. Espinós, J. Morales, A. Barranco , A. Caballero , J. P. Holgado y A. R. González-Elipe. "Interface Effects for $\mathrm{Cu}, \mathrm{CuO}$, and $\mathrm{Cu} 2 \mathrm{O}$ Deposited on $\mathrm{SiO} 2$ and $\mathrm{ZrO} 2$. XPS Determination of the Valence State of Copper in $\mathrm{Cu} / \mathrm{SiO} 2$ and $\mathrm{Cu} / \mathrm{ZrO} 2$ Catalysts", The journal Physical Chemistry, vol. 106, no. 22, pp. 6921-6929, 2002.
Enero - Junio 2016 ISSN 0122-820X

E-ISSN 2422-5053

PP: $89-96$ 
No. 1

Enero - Junio 2016

ISSN 0122-820X

E-ISSN 2422-5053

PP: 89-96
[6] C. M. Pelicano, J. C. Felizco y M. D. Balela. "Formation of copper oxide nanoestructures by solution -phase method for antibacterial applications", Advanced Materials, structures and Mechanical Engineering, Edit. Mosbeh Kaloop, pp. 203, 2015.

[7] Q. Yong, Y. Fucheng, X. Jianping, L. Zhang-Gao. "Synthesis of Cuprous Composite with an Excellent Electrocatalytic Activity Towards Glucose", International Journal of electroquimico Science, vol. 7, pp. 10063-10073, 2012.

[8] W. Zhou, B. Yan, C. Cheng, C. Cong, H. Hu, H. F. y T Yu. "Facile synthesis and shape evolution of highly symmetric 26-facet polyhedral microcrystals of Cu2O", CrystEngComm, no. 11, pp. 2291-2296, 2009.

[9] Y. Wang, D. Huang,X. Zhu,Y. Ma,H Geng, Y. Wang, G. Yin,D. He, Z.Yang. "Surfactant-free synthesis of $\mathrm{Cu} 2 \mathrm{O}$ hollow spheres and their wavelengthdependent visible photocatalytic activities using LED lamps as cold light sources", Nanoscale Research Letterst, vol. 9, no. 624, 2014.

[10] W.Wang, O Varghese,C. Ruan. "Synthesis of $\mathrm{CuO}$ and $\mathrm{Cu} 2 \mathrm{O}$ crystalline nanowires using $\mathrm{Cu}(\mathrm{OH}) 2$ nanowire templates", Journal of Materials Research, vol. 18, no. 2, pp. 2756-2759, 2003.

[11] J.Hernandez,P. Wrschka, G.S. Oehrlein. "Surface Chemistry Studies of Copper Chemical Mechanical Planarization", Journal of The Electrochemical Society, vol. 148, no. 7, pp. G389-G397, 2001.

[12] F. C. S. Paschoalino, M. P. Paschoalino, E. Jordão, W. F. Jardim. "Evaluation of $\mathrm{TiO} 2, \mathrm{ZnO}, \mathrm{CuO}$ and $\mathrm{Ga} 2 \mathrm{O} 3$ on the Photocatalytic Degradation of Phenol
Using an Annular-Flow". Open Journal of Physical Chemistry, vol. 2, no.3, pp. 135-140, 2012.

[13] N. M. Julkapli, S. Bagheri, S. B. A. Hamid. "Recent Advances in HeterogeneousPhotocatalytic Decolorization of Synthetic Dyes", The Scientific World Journal, vol. 2014, pp. 25, 2014.

[15] A. O. Ibhadon, P. Fitzpatrick. "Eeterogeneous Photocatalysis: Recent Advances and Applications", Catalysts, vol. 3, no. 1, 189-218, 2013.

[15] A. M. Hasna. "Reduction of NOX Gases Using Copper Zeolite Catalyst, Proceedings of the World" Congress on Engineering, London, U.K., vol. 1, 2009.

[16] A. M. Raba, J-Barba-Ortega, M. R. Joya, "The effect of the preparation method of Nb2O5oxide influences the performance of the photocatalytic activity", Applied Physics A, vol. 119, no. 3, 923-928, 2015. 